\title{
Electrically Conductive Silicone-based Nanocomposites Incorporated with Carbon Nanotube and Silver Nanowire for Stretchable Electrodes
}

Tae Gon Kim ${ }^{1}$, Huyn Sik Eom ${ }^{1}$, Jong Hwi Kim ${ }^{1,2}$, Jik Kyo Jung ${ }^{2}$, Keon-Soo Jang ${ }^{1, *}$, Seong Jae Lee ${ }^{1, *}$

${ }^{1}$ Department of Polymer Engineering, School of Chemical and Materials Engineering, The University of Suwon, Hwaseong, Gyeonggi 18323, Republic of Korea

${ }^{2}$ NanoChemTech Inc., 112 Yangseong-ro, Yangseong-myeon, Anseong, Gyeonggi 17502, Republic of Korea 


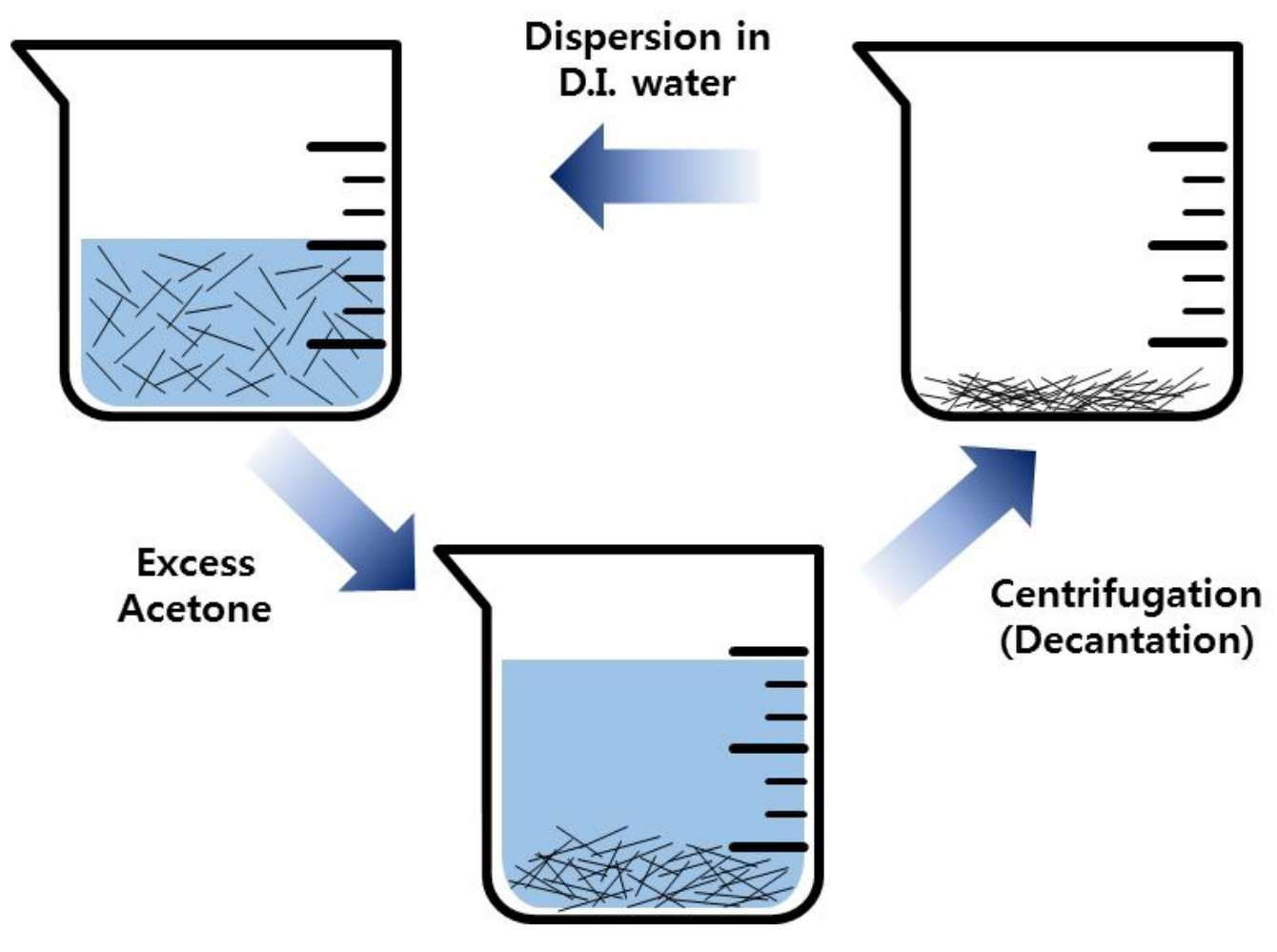

Figure S1. Schematic diagram illustrating the purification process of AgNW. 
(a)
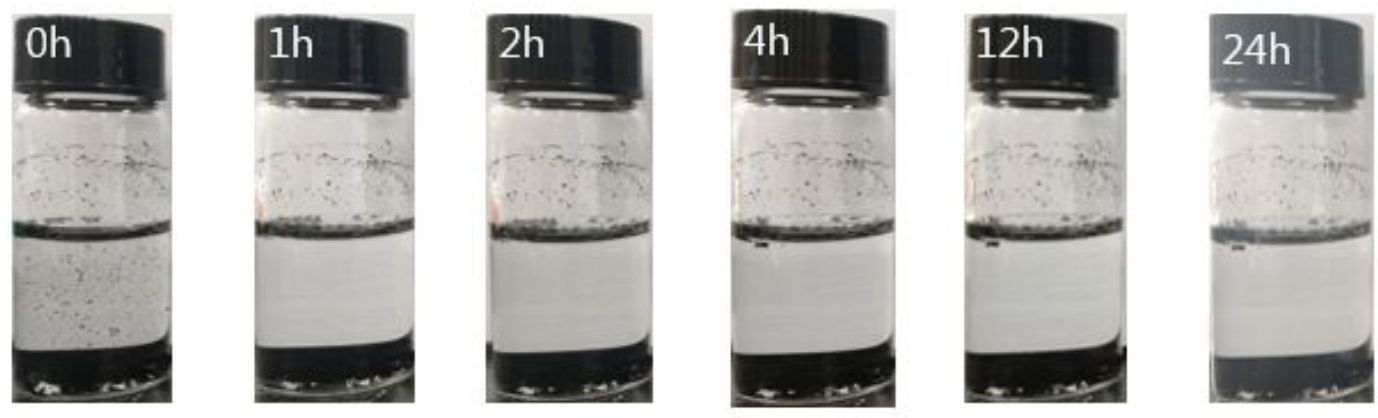

(b)
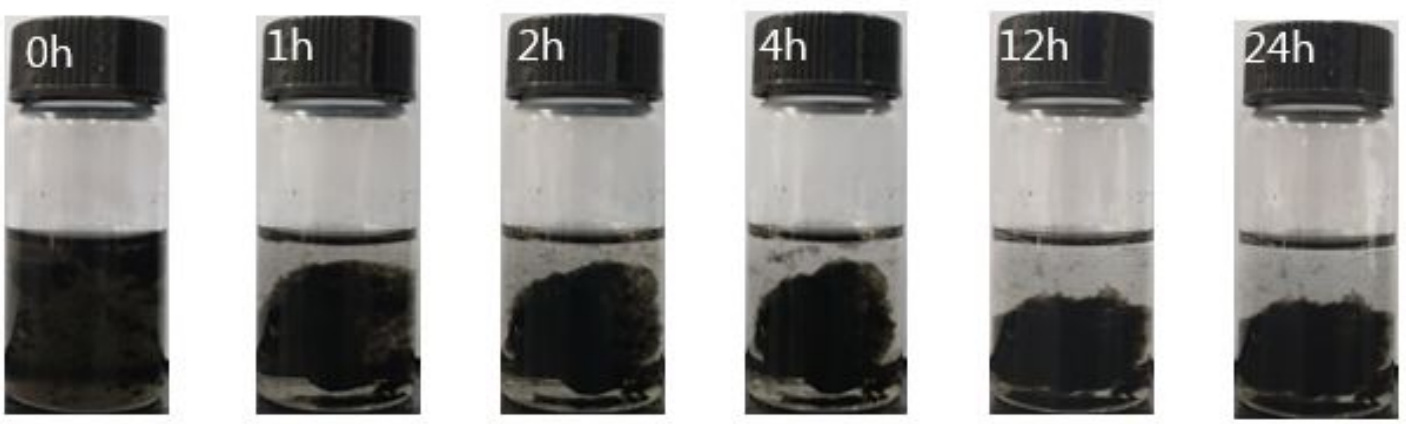

(c)
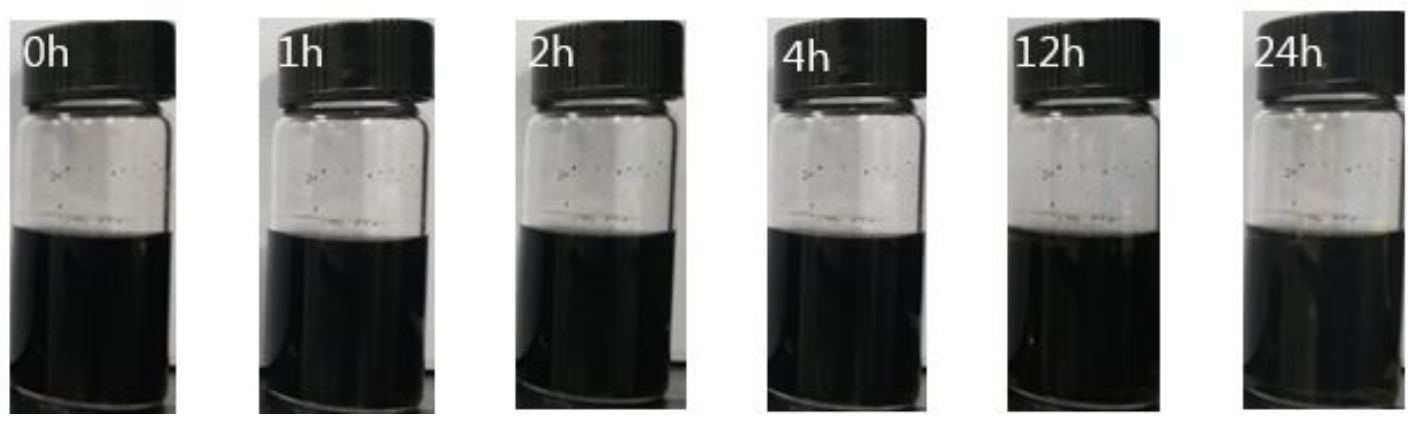

Figure S2. Dispersion stability of CNT in different solvent: (a) Water, (b) ethylene glycol, and (c) IPA. 


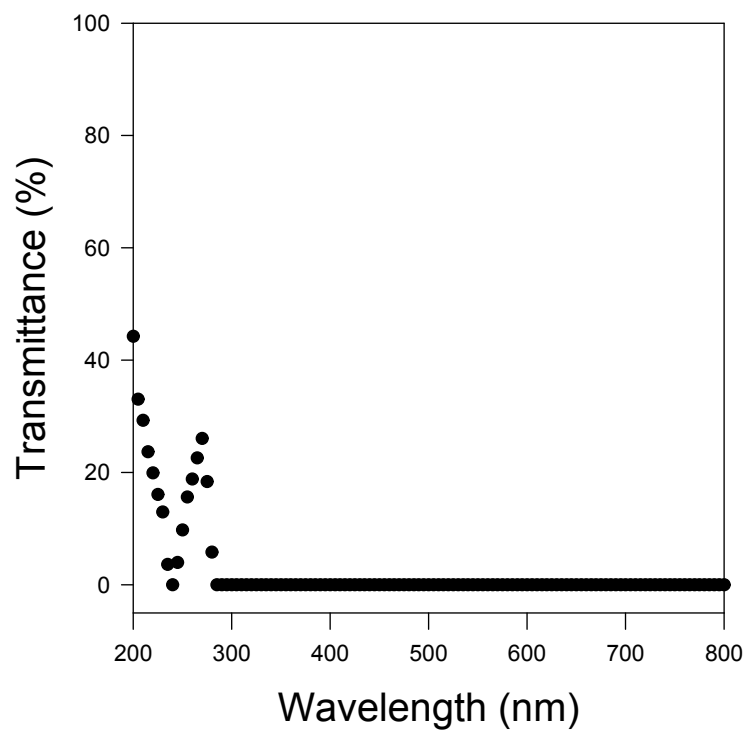

Figure S3. Dispersion of PVP-capped AgNW in IPA. 

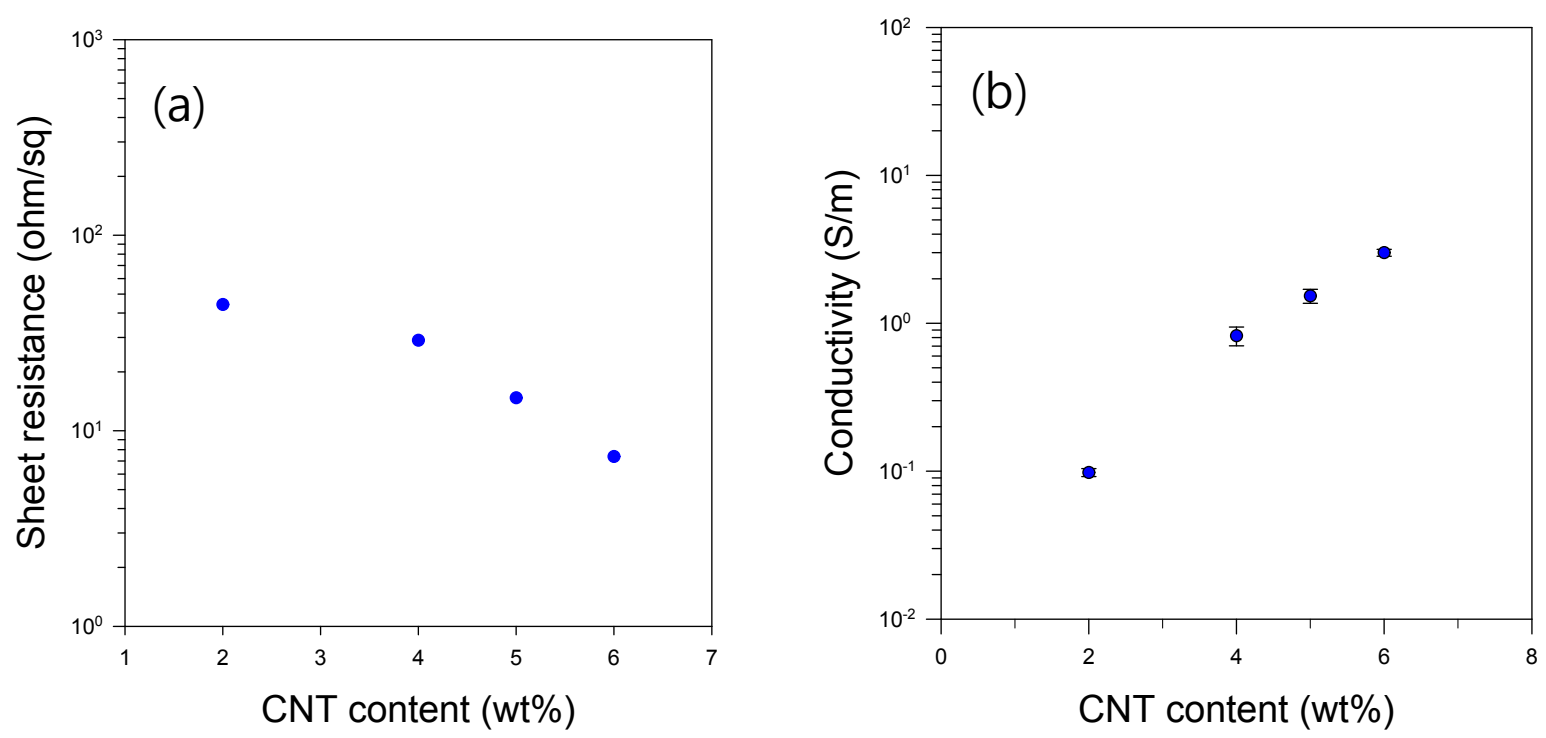

Figure S4. Electrical properties of PDMS/CNT nanocomposites as a function of CNT content: (a) Sheet resistance and (b) electrical conductivity. 


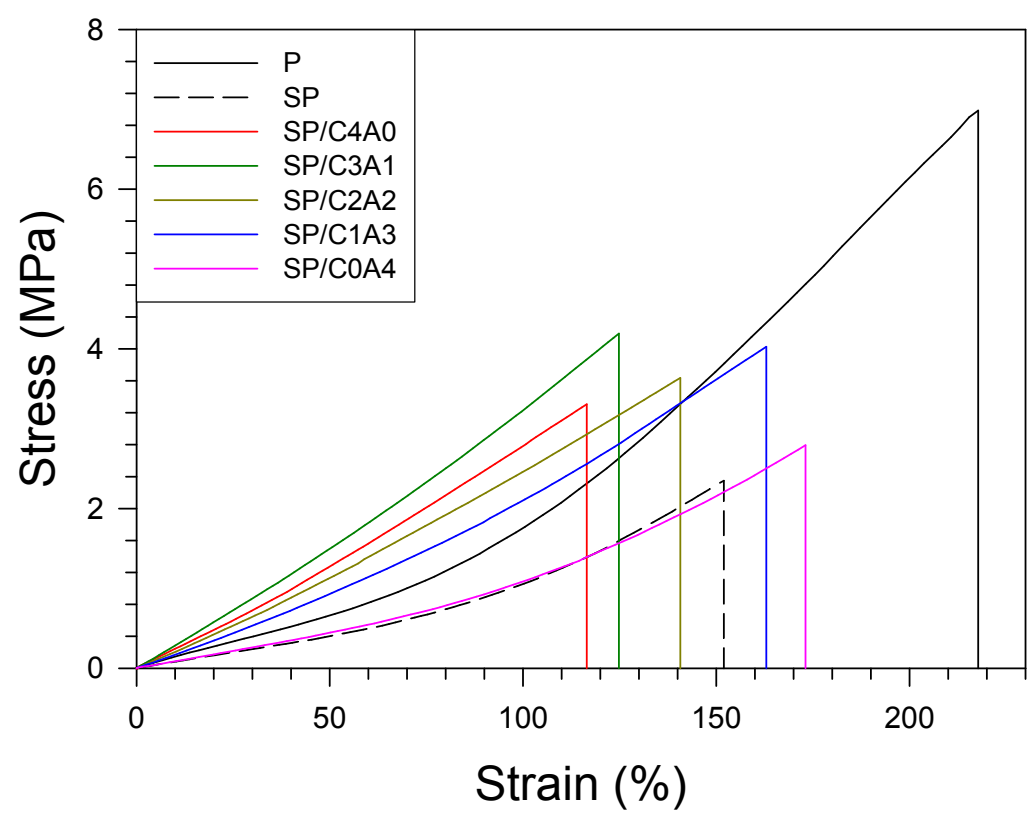

Figure S5. Stress-strain curves (tensile mode) of PDMS/CNT/AgNW nanocomposites with different $\mathrm{CNT} / \mathrm{AgNW}$ ratios (wt $\% / w t \%)$. 


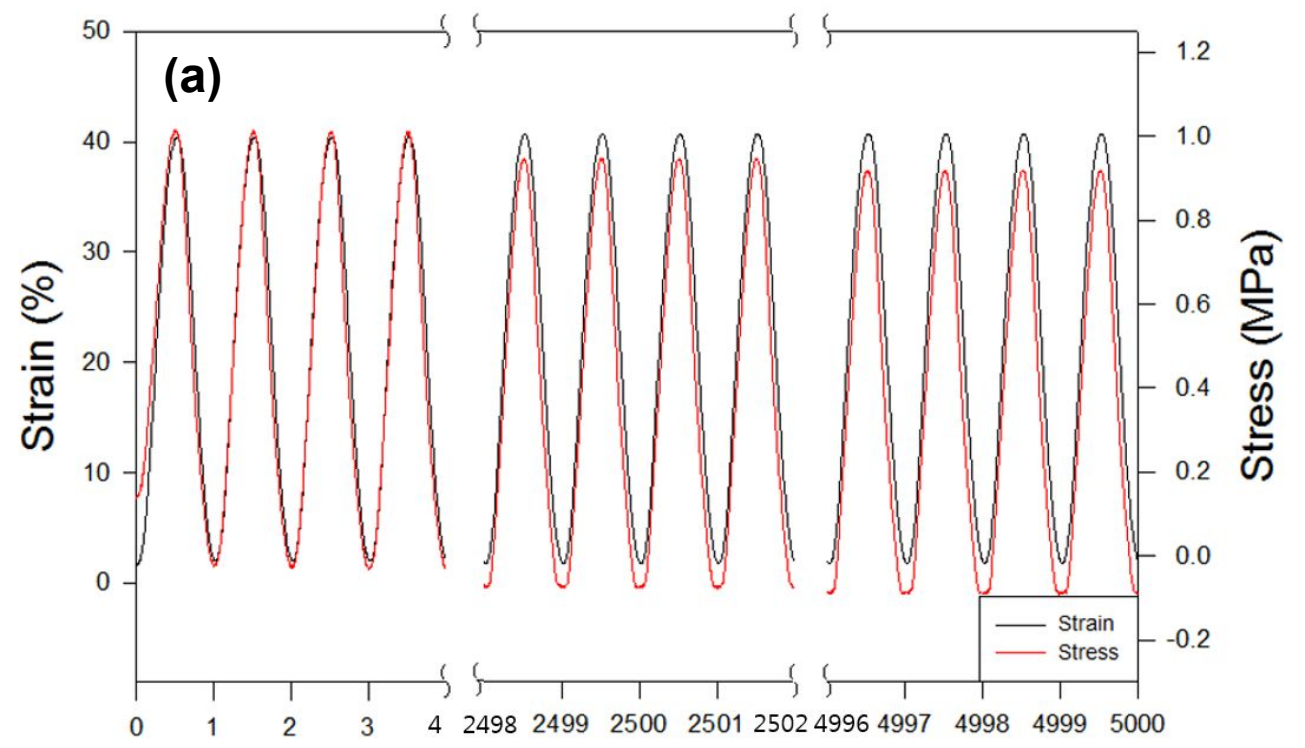

No. of cycles

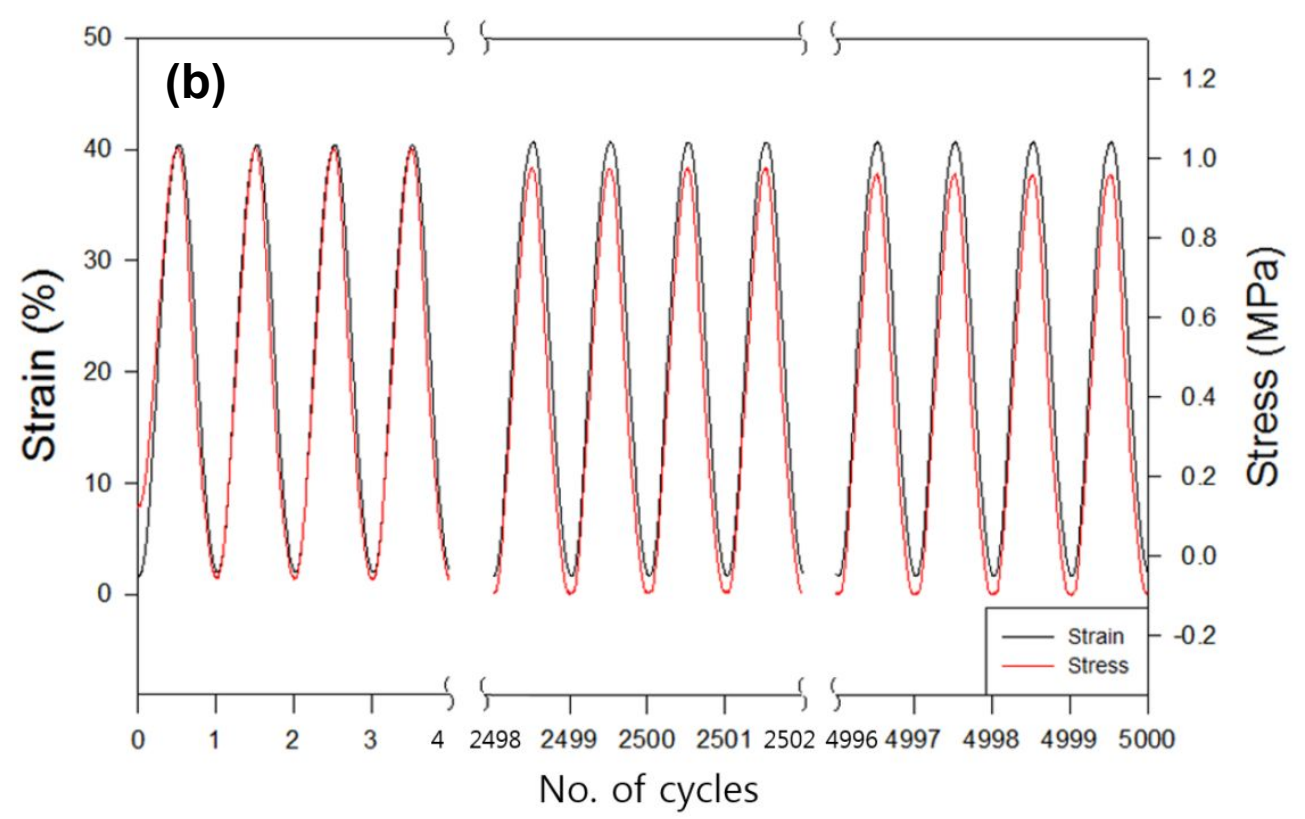



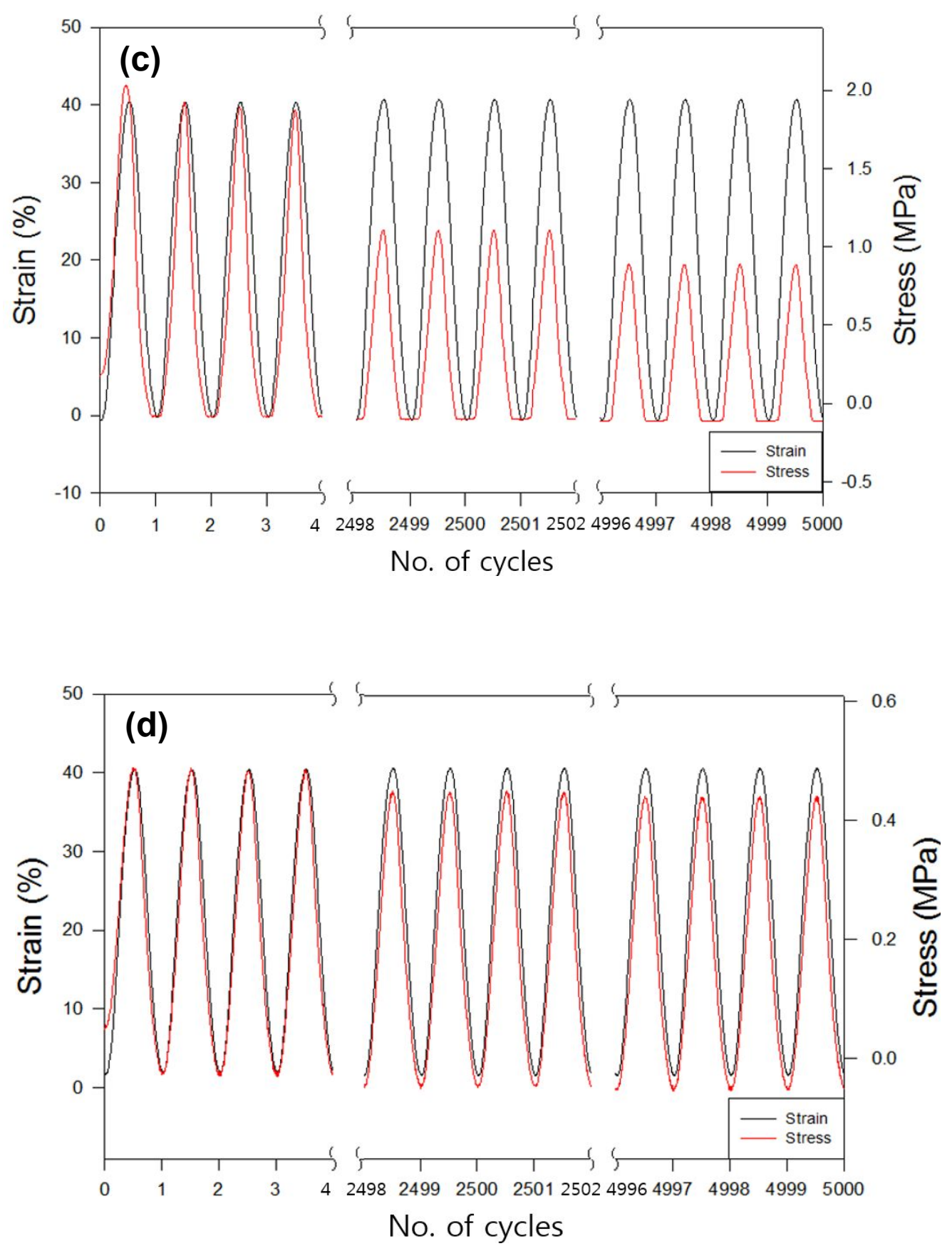

Figure S6. Dynamic properties of PDMS/CNT/AgNW with different CNT/AgNW ratios (wt\%/wt\%) during cyclic strain tests: (a) P, (b) SP, (c) SP/C4/A0, and (d) SP/C0/A4. 

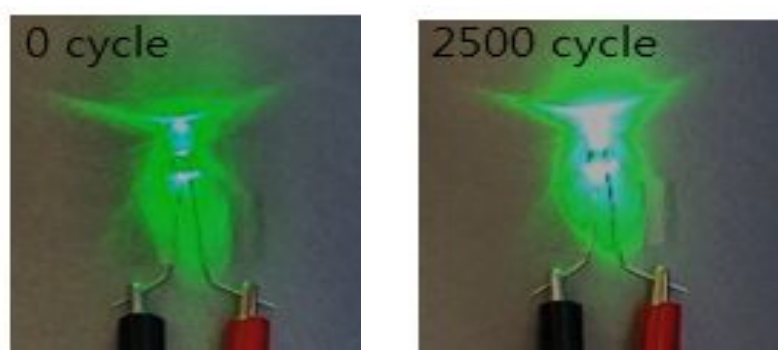

(a)
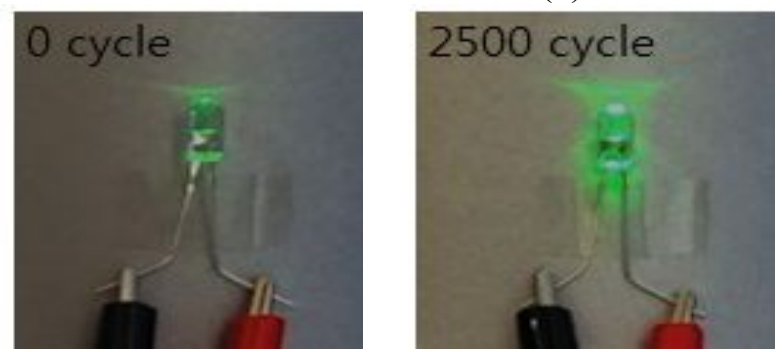

(b)
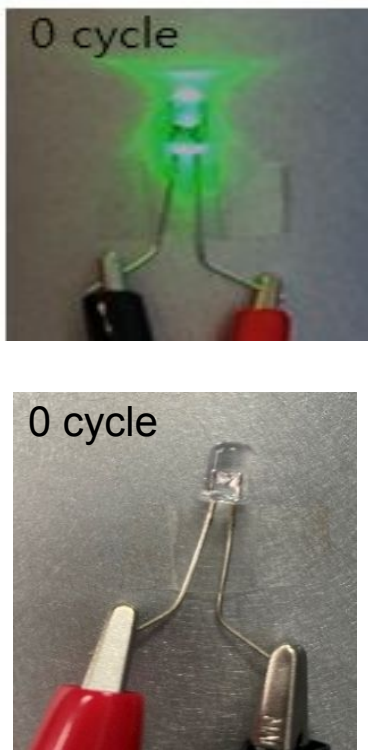

(c)

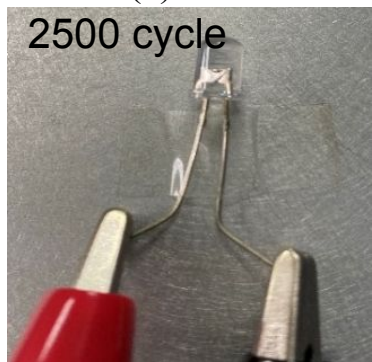

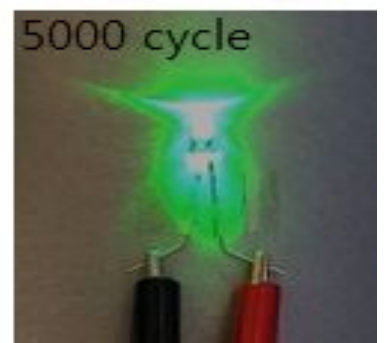
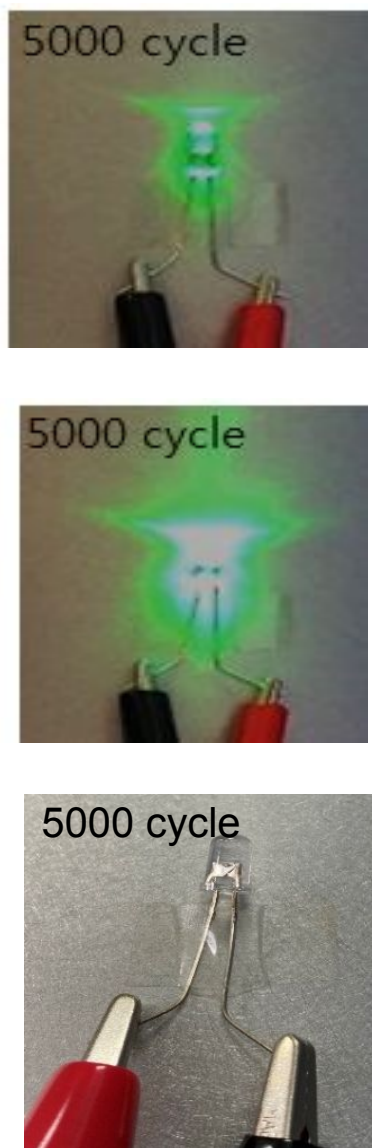

(d)

Figure S7. Change of LED light according to cycle: (a) SP/C4-A0 (b) SP/C3.5-A0.5

(c) SP/C2.5/A1.5 (d) SP/C0/A4 
Table S1. Tensile properties of PDMS/CNT nanocomposites as a function of CNT content

\begin{tabular}{cccc}
\hline & $\begin{array}{c}\text { Tensile strength } \\
(\mathrm{MPa})\end{array}$ & $\begin{array}{c}\text { Elongation at break } \\
(\%)\end{array}$ & $\begin{array}{c}\text { Young's modulus } \\
(\mathrm{MPa})\end{array}$ \\
\hline CNT 2\% & $3.01 \pm 0.2$ & $180.4 \pm 0.6$ & $1.11 \pm 0.12$ \\
CNT 4\% & $3.51 \pm 0.2$ & $116 \pm 0.78$ & $2.64 \pm 0.18$ \\
CNT 5\% & $2.46 \pm 0.2$ & $107.1 \pm 11.3$ & $2.72 \pm 0.4$ \\
CNT 6\% & $2.08 \pm 0.5$ & $77.02 \pm 10.2$ & $3.09 \pm 0.21$ \\
\hline
\end{tabular}

Table S2. Tensile properties of PDMS/CNT/AgNW nanocomposites

\begin{tabular}{cccccccccc}
\hline $\begin{array}{c}\text { Mechanical } \\
\text { properties }\end{array}$ & PDMS & $\begin{array}{c}\text { PDMS }+ \\
\text { Silicone } \\
\text { oil }\end{array}$ & $\begin{array}{c}\text { C4- } \\
\text { A0 }\end{array}$ & $\begin{array}{c}\text { C3.5- } \\
\text { A0.5 }\end{array}$ & $\begin{array}{c}\text { C3- } \\
\text { A1 }\end{array}$ & $\begin{array}{c}\text { C2.5- } \\
\text { A1.5 }\end{array}$ & $\begin{array}{c}\text { C2- } \\
\text { A2 }\end{array}$ & $\begin{array}{c}\text { C1- } \\
\text { A3 }\end{array}$ & $\begin{array}{c}\text { C0- } \\
\text { A4 }\end{array}$ \\
\hline Tensile & 7.4 & 2.3 & 3.5 & 3.6 & 4 & 3.7 & 3.4 & 4.1 & 2.8 \\
strength & \pm & \pm & \pm & \pm & \pm & \pm & \pm & \pm & \pm \\
(MPa) & 0.7 & 0.3 & 0.2 & 0.3 & 0.3 & 0.4 & 0.3 & 0.2 & 0.1 \\
Elongation & 225 & 144 & 116 & 123 & 123 & 148 & 147 & 160 & 172 \\
at break & \pm & \pm & \pm & \pm & \pm & \pm & \pm & \pm & \pm \\
(\%) & 18.5 & 9.0 & 0.8 & 1.5 & 7.1 & 9.2 & 13.6 & 6.2 & 7.4 \\
Young's & 1.5 & 0.8 & 2.6 & 2.3 & 2.6 & 2.1 & 1.7 & 1.7 & 0.9 \\
modulus & \pm & \pm & \pm & \pm & \pm & \pm & \pm & \pm & \pm \\
(MPa) & 0.01 & 0.02 & 0.2 & 0.1 & 0.2 & 0.1 & 0.3 & 0.2 & 0.04 \\
\hline
\end{tabular}

